radiotherapy was required for mass lesions in 3 patients followed for a mean time of 2 to 3 years. (DiMario FJ Jr, Ramsby G. Arch Neurol 1998;55:500-505).

\title{
PREFRONTAL CORTICAL ASYMMETRY AND ATTENTION DEFICIT HYPERACTIVITY DISORDER
}

Fractal information dimension (FID), a scale-free measure, was used to assess the prefrontal cortical convolution complexity and asymmetry in 12 boys with ADHD and 11 controls, in an MRI study at the Chinese Academy of Sciences, and other centers in Beijing, PR China. A left-greater-than-right prefrontal cortical convolution complexity was present in both groups, but in ADHD patients the complexity pattern was significantly reduced. This resulted in a significant reduction of the normal prefrontal cortical asymmetry pattern in ADHD compared to control subjects. (Li X, Jiang J, Zhu W et al. Asymmetry of prefrontal cortical convolution complexity in males with attention-deficit/hyperactivity disorder using fractal information dimension. Brain Dev Nov 2007;29:649-655). (Respond: E-mail: jiangtz(onlpr.ia.ac.cn (T Jiang)).

COMMENT. MRI volumetric studies have shown prefrontal cortical abnormalities in ADHD, sometimes affecting the right prefrontal and other brain regions. (Castellanos FX et al. Arch Gen Psychiatry 1996;53:607-616). The current study assesses the cortical convolution (or gyrification) complexity by using the fractal information dimension, a scalefree measure unaffected by variations in volume of the brain region. A greater FID value shows a higher cortical convolution level of cerebral sulci and gyri. The study found a significantly reduced cortical convolution complexity in the left prefrontal region in ADHD boys. Other studies have found volume reduction in the left prefrontal cortex in children and adults with ADHD, and reduced left prefrontal cortical thickness and cerebral blood flow in ADHD children. The findings indicate an underdevelopment of the left prefrontal lobe in ADHD, in support of an organic neurological basis for the syndrome. A frontal-motor cortex disconnection syndrome, or 'lazy' frontal lobe, in ADHD is one hypothesis, resulting in failure of the frontal lobe to inhibit excessive motor activity and sustain attention. (Niedermeyer E, Naidu SB. Clin Electroencephalogr 1997;28:130-136). The frontal lobe stimulant effect of methylphenidate corrects the impaired motor inhibition.

Acute dyskinesia on starting methylphenidate after risperidone withdrawal is reported in a 7-year-old boy with conduct disorder and ADHD (Hollis CP et al. Pediatr Neurol Oct 2007;37:287-288). Within 8 hours of taking Concerta $36 \mathrm{mg}$, the boy manifested twitching of hands and feet, throat clearing, overactivity, and vomiting. After risperidone reinstatement $1.5 \mathrm{mg}$, tics resolved within a few hours. A second attempt to substitute Concerta $18 \mathrm{mg}$ for risperidone, 5 months later, resulted in almost immediate mouthing movements, tongue protrusion, shoulder shrugging, and dystonic flexion of wrist and fingers. Movements resolved by the following morning, and a trial of atomoxetine provided adequate control of ADHD and aggressive behavior. The authors advise caution in the use of combinations of risperidone and psychostimulants. Slow neuroleptic withdrawal and extended washout period should precede initiation of stimulant medication in low dose. 